\title{
PI3K signaling of autophagy is required for starvation tolerance and virulence of Cryptococcus neoformans
}

\author{
Guowu Hu, ${ }^{1}$ Moshe Hacham, ${ }^{1}$ Scott R. Waterman, ${ }^{1}$ John Panepinto, ${ }^{1}$ Soowan Shin, ${ }^{1}$ \\ Xiaoguang Liu, ${ }^{1}$ Jack Gibbons, ${ }^{2}$ Tibor Valyi-Nagy, ${ }^{3}$ Keisuke Obara, ${ }^{4}$ H. Ari Jaffe, ${ }^{5}$ \\ Yoshinori Ohsumi, ${ }^{4}$ and Peter R. Williamson ${ }^{1,3,6}$
}

\begin{abstract}
${ }^{1}$ Section of Infectious Diseases, Department of Medicine, ${ }^{2}$ Department of Biological Sciences, and ${ }^{3}$ Department of Pathology, Department of Medicine, University of Illinois at Chicago, Chicago, Illinois, USA. ${ }^{4}$ Division of Molecular Cell Biology, National Institute for Basic Biology, Okazaki, Japan. ${ }^{5}$ Section of Pulmonary Medicine and Critical Care, Department of Medicine, University of Illinois at Chicago, Chicago, Illinois, USA. ${ }^{6}$ Jesse Brown VA Medical Center, Chicago, Illinois, USA
\end{abstract}

\begin{abstract}
Autophagy is a process by which cells recycle cytoplasm and defective organelles during stress situations such as nutrient starvation. It can also be used by host cells as an immune defense mechanism to eliminate infectious pathogens. Here we describe the use of autophagy as a survival mechanism and virulence-associated trait by the human fungal pathogen Cryptococcus neoformans. We report that a mutant form of C. neoformans lacking the Vps34 PI3K ( $v$ ps 34 4 ), which is known to be involved in autophagy in ascomycete yeast, was defective in the formation of autophagy-related 8-labeled (Atg8-labeled) vesicles and showed a dramatic attenuation in virulence in mouse models of infection. In addition, autophagic vesicles were observed in WT but not $\nu p s 34 \Delta$ cells after phagocytosis by a murine macrophage cell line, and Atg8 expression was exhibited in WT C. neoformans during human infection of brain. To dissect the contribution of defective autophagy in $\nu p s 34 \Delta$ C. neoformans during pathogenesis, a strain of $C$. neoformans in which Atg8 expression was knocked down by RNA interference was constructed and these fungi also demonstrated markedly attenuated virulence in a mouse model of infection. These results demonstrated PI3K signaling and autophagy as a virulence-associated trait and survival mechanism during infection with a fungal pathogen. Moreover, the data show that molecular dissection of such pathogen stress-response pathways may identify new approaches for chemotherapeutic interventions.
\end{abstract}

\section{Introduction}

Cryptococcus neoformans ( $\mathrm{Cn}$ ) is a major fungal pathogen primarily infecting immunocompromised hosts, especially patients with impaired cellular immunity related to HIV infection (1). It is generally believed that inhalation of desiccated yeasts or basidiospores from the environment is the route for primary pulmonary $\mathrm{Cn}$ infection. The alveolar macrophages (AMs) therefore serve as one of the first lines of anticryptococcal defense that must be overcome by a successful fungal intruder (2). While much is known about specific immunological killing mechanisms of macrophages that result in containment of intracellular yeast cells (3), only recently has study been devoted to understanding the host's ability to deprive the pathogen of essential elements necessary for survival and virulence. For example, in cryptococcosis, deprivation of oxygen and glucose results in a requirement for the oxygen sensor, Sre1 (4), and the gluconeogenesis enzyme Pck1 (5) in the pathogen. Encountering such a hostile nutrient environment thus requires a specific programmatic response by the pathogen, dissection of which may yield new insights into unique attributes of the host-pathogen interaction. Such study may also contribute to our understanding of how organisms adapt during their transition from environmental saprophytes to pathogens and may provide genetic predictors of strains that

Nonstandard abbreviations used: AM, alveolar macrophage; Cn, Cryptococcus neoformans; PI3P, phosphatidylinositol 3 phosphate; Sc, Saccharomyces cerevisiae. Conflict of interest: The authors have declared that no conflict of interest exists. Citation for this article: J. Clin. Invest. 118:1186-1197 (2008). doi:10.1172/JCI32053. have evolved to become the more successful pathogens. For example, clinical isolates showing more effective induction of high-affinity copper transport were recently found to be associated with a propensity to cause more severe neurological disease in a cohort of transplant patients (6).

The class III PI3K in ascomycete yeast, Vps34, has been shown to be involved in vesicular transport of vacuolar hydrolases to the vacuole lumen via endosomes (the so-called "CPY pathway") and autophagy under nutrient-deprived conditions (7). CPY requires the formation of PI3K complex II composed of Vps34, Vps15, Vps30 (Atg6), and Vps38 (8). Autophagy, involved in nutrient conservation during starvation, requires formation of a type I kinase subcomplex composed of Vps34, Vps15, Atg14, and Atg6 $(8,9)$, leading to transport vesicle formation initiated by lipid binding (lipidation) of Atg8 (10). The Tor signaling pathway in yeast responds to nutrient levels, and inhibition by rapamycin leads to an artificial induction of autophagy under nutrient-replete conditions (11). In mammalian systems, an additional class I PI3K activity is associated with activation of Tor signaling through a TSC2/TSC1-dependent pathway and is inhibitable by nanomolar concentrations of wortmannin (12). Indeed, while there is no TSC2/TSC1 homolog in Saccharomyces cerevisiae (Sc), a homolog of TSC2 has been described in Schizosaccharomyces pombe (13), and a homolog in $\mathrm{Cn}$ is apparent in the UniProt genome database (http://beta.uniprot.org/; accession no. Q5KJR4; 51\% identical to S. pombe), suggesting higher yeast species may share a relationship between PI3K activity and TSCmediated Tor activity. 
A
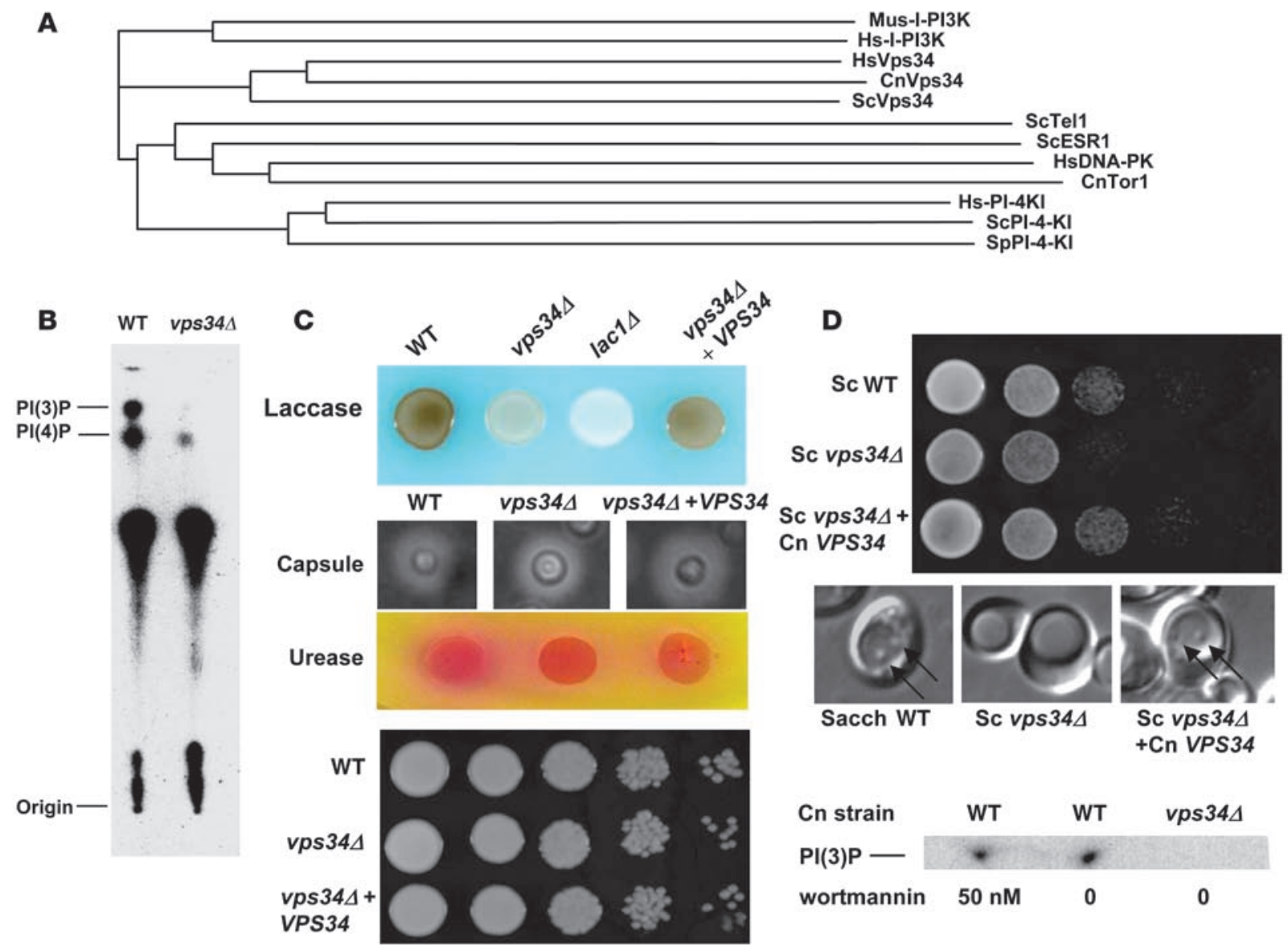

Sacch WT

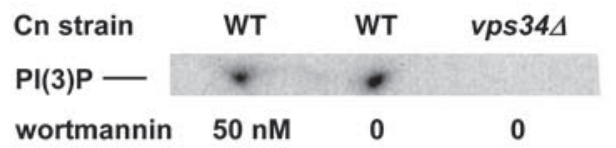

\section{Figure 1}

Phenotypes of a cryptococcal VPS34 homolog in vitro. (A) Clustal-W analysis comparing closely related kinases. Mus musculus class I PI3K (GenBank NP 032865); human class I PI3K, Vps34, DNA-PK, and PI4K (GenBank AAX41023, NP 002638, AAA79184, and AAA56839, respectively); Sc Tel1, Esr1, PI4KI, Vps34 (GenBank CAA84909, CAA85094, NP 013408, and CAA37610, respectively); S. pombe PI4K (GenBank NP 595304); and Cn Tor1 (GenBank AAD16273). (B) Indicated mid-log phase fungal cells were starved for $4 \mathrm{~h}$ in YNB without amino acids or ammonium sulfate and cell lysates assayed for PI kinase activity by autoradiography of thin-layer chromatograms, as described in Methods. (C) Indicated $\mathrm{Cn}$ cells were assayed for laccase by melanin formation, capsule by India Ink microscopy, urease activity, and growth at $37^{\circ} \mathrm{C}$ on YPD agar as described in Methods. (D) Indicated Sc mid-log phase cells were assayed for growth at $37^{\circ} \mathrm{C}$ in YPD (upper panel) or incubated in YNB without amino acids or ammonium sulfate for $4 \mathrm{~h}$ in the presence of $1 \mathrm{mM}$ PMSF and observed using DIC microscopy or as described in Methods (middle panel). Arrows point to autophagic bodies. Lower panel: Cn cells were subjected to starvation conditions and assayed for PI kinase activity as in $\mathbf{B}$ with or without addition of $50 \mathrm{nM}$ wortmannin.

In the present study, we report the targeted deletion of a cryptococcal VPS34 PI3K gene linked to intact laccase expression and use this to identify genetically linked attributes of virulence. The results show that although VPS34 was largely dispensable for growth of $\mathrm{Cn}$ in nutrient-replete conditions at $37^{\circ} \mathrm{C}$ and was not required for expression of several important virulence factors such as capsule and urease, it played a large role in virulence and survival in macrophages. In addition, the VPS34 mutation was associated with a defect in tolerance to nutrient starvation, related to an inability to undergo autophagy. Furthermore, molecular dissection of VPS34-related phenotypes was undertaken to determine the contribution of autophagy in cryptococcal pathogenesis, with the conclusion that RNAi suppression of the autophagy-related ATG8 gene also resulted in loss of virulence in 2 mouse models of cryptococcosis. These studies thus provide genetic support for a role for PI3K and autophagy in the adaptation to nutrient starvation and virulence for a pathogenic fungus.

\section{Results}

Identification and mutation of a VPS34 homolog in Cn. Analysis of a laccase-associated cryptococcal annotated gene sequence (TIGR Database locus name 186.m03746; http://www.tigr.org/tdb/ e2k1/cna1) showed strong homology to PI3Ks, including VPS34 from Sc (35\% identity; E value, e-130), and contained 2 conserved PI3K sequences, a catalytic domain (PI3Kc) at aa 554-918 (E value, 2e-124), and an accessory domain (PI3Ka) at aa 344-527 (E value, $6 \mathrm{e}-48$ ). BLAST analysis of the NCBI database showed homology with PI3Ks only within the first 200 matches. In addition, Clustal-W analysis (EMBL-EBI database; http://www.ebi.ac.uk/Tools/ clustalw/index.html) comparing closely related kinase members, as described by Shelton et al. (14), showed highest similarity to the class III Vps34 class of PI3Ks exemplified by Vps34 of Sc, less homology with the class I PI3Ks such as those from mice or humans, and even less similarity to other related kinases (Figure 1A). A Cn knockout strain, vps34A, was constructed (Supplemental 

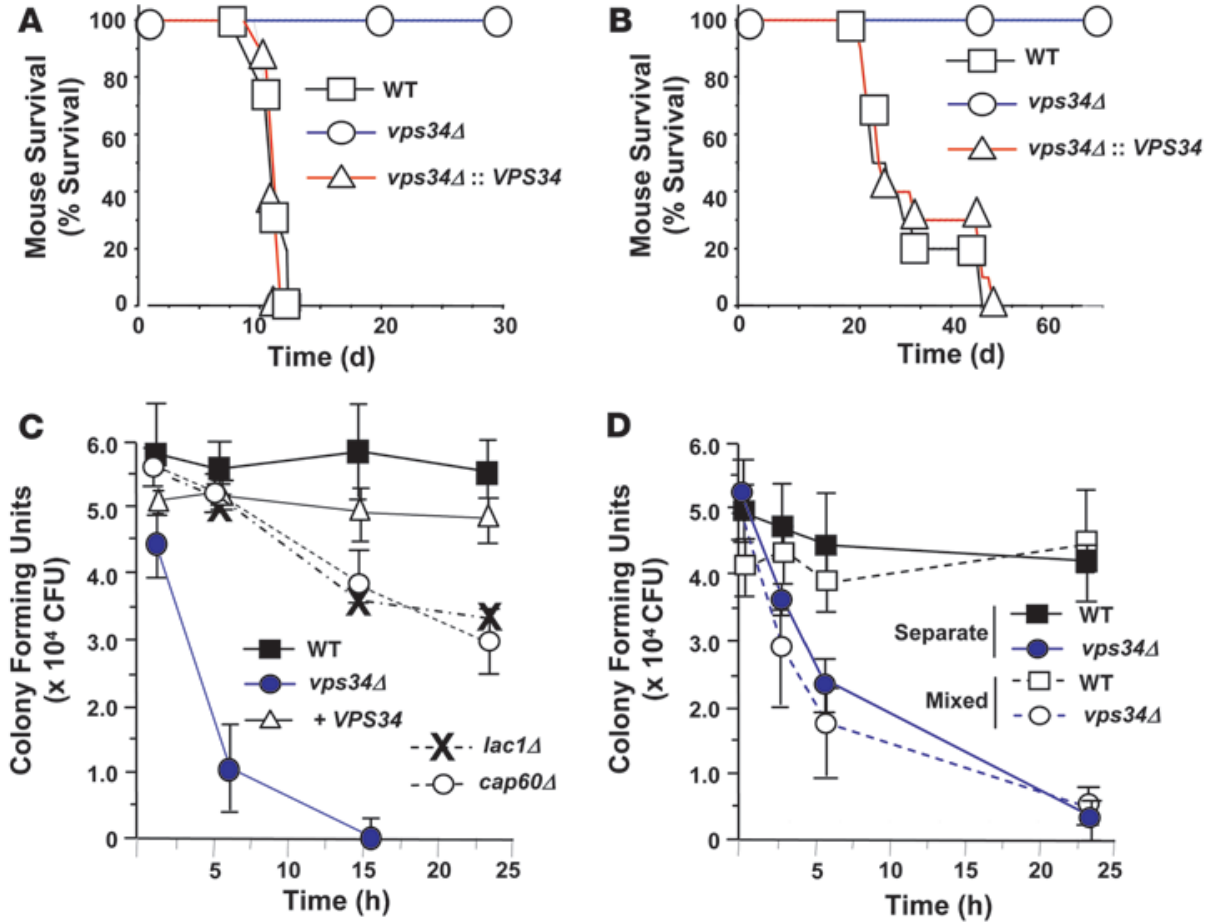

\section{Figure 2}

Role of VPS34 in cryptococcosis. Indicated strains were inoculated intravenously into 10 Swiss Albino mice (106 CFU; A) or intranasally into $10 \mathrm{CBA} / \mathrm{J}$ mice (105 CFU; $B$ ) and sacrificed when moribund. (C) Same as in B, but animals were sacrificed at the indicated times, lungs harvested, and fungal cells measured by CFU on YPD (values represented as $\pm \mathrm{SEM} ; n=3$ for each strain at each time point) (D) Same as in C except that mice were inoculated with either $10^{5}$ CFU of the indicated strains or a mixture of $10^{5} \mathrm{WT}$ and $10^{5} \mathrm{vps} 34 \Delta$ mutant strains in PBS containing $1.0 \%$ glucose, and cells were recovered from lungs and fungal strains identified in mixtures, as described in Methods.
Figure 1; supplemental material available online with this article; doi:10.1172/JCI32053DS1) that demonstrated production of a number of phosphorylated phosphatidylinositol phosphates, including phosphatidylinositol 4 phosphate (PI4P), as described in ascomycete yeast (15), but also showed defective production of PI3P (Figure 1B) and reduced expression of laccase. However, the mutant retained production of the virulence factors capsule and urease (Figure 1C). Growth rate of the cryptococcal $v p s 34 \Delta$ mutant was similar to WT at $30^{\circ} \mathrm{C}$ on agar (data not shown) and in liquid culture (doubling times: WT, $2.6 \pm 0.3 \mathrm{~h}$; vps $34 \Delta, 2.8 \pm 0.3 \mathrm{~h}$; $v$ ps $34 \Delta+V P S 34,2.4 \pm 0.3 \mathrm{~h}$ ), but there was only a barely discernible difference at $37^{\circ} \mathrm{C}$ on agar (Figure $1 \mathrm{C}$ ) or in liquid culture (doubling times: WT, $2.8 \pm 0.3 \mathrm{~h} ; v p s 34 \Delta, 3.5 \pm 0.4 \mathrm{~h} ; v p s 34 \Delta+V P S 34,2.6 \pm 0.3 \mathrm{~h}$; $P<0.05 v p s 34 \Delta$ vs. WT or $v p s 34 \Delta+V P S 34)$. Retention of near WT growth at $37^{\circ} \mathrm{C}$ is in contrast to a more severe temperature-dependent growth defect of the homolog mutant strain from Sc (16). This difference could be due to redundancy in growth pathways in the basidiomycetous fungi and has also been found with other vps-class mutants from this fungus (17). To further characterize the cryptococcal Vps34 activity, a Sc vps34 4 mutant was complemented with a cDNA fragment of the cryptococcal gene expressed under a Saccharomyces constitutive promoter. As shown in Figure $1 \mathrm{D}$, complementation of the Sc vps $34 \Delta$ mutant with Cn VPS34 resulted in restoration of its temperature-sensitive defect at $37^{\circ} \mathrm{C}$ as well as production of autophagic bodies under starvation conditions (WT, $3.6 \pm 0.3$; Sc vps34 $0.1 \pm 0.1$; Sc vps34 0. Cn VPS34, $2.3 \pm 0.4$ vacuolar vesicles per cell; $n=15, P<0.0001$, vps $34 \Delta$ vs. either WT or Sc vps $34 \Delta+$ Cn VPS34) that were freely mobile within the Sc vacuole (data not shown). Furthermore, WT cryptococcal cell PI3P production was resistant to nanomolar concentrations of wortmannin (Figure 1D), consistent with a class III PI3K activity (15).

Role of PI3K in cryptococcal pathogenesis. Because of retention of growth at $37^{\circ} \mathrm{C}$ in the cryptococcal vps $34 \Delta$ mutant strain, we tested for virulence in mice. While some attenuation in virulence was expected because of the minimally reduced growth rate and reduced laccase expression, the vps $34 \Delta$ mutant strain was found to be avirulent in an intravenous (Figure $2 \mathrm{~A}$ ) as well as an intranasal (Figure 2B) mouse model and organ culture of surviving mice failed to identify live fungal cells in brain or lung. Parallel experiments comparing virulence of a lac $1 \Delta$ mutant with WT in the same genetic background and using the same models and fungal inoculum found a smaller reduction in virulence in the lac1 $\Delta$ mutant; (intravenous, Swiss Albino: median survival, WT, $7 \mathrm{~d}, \operatorname{lac} 1 \Delta, 11 \mathrm{~d}$, $P<0.05$; intranasal, CBA/J: median survival, WT, $25 \mathrm{~d}$, lac1 $1 \Delta, 29 \mathrm{~d}$, $P<0.05)$, suggesting that the avirulence of the $v p s 34 \Delta$ mutant was not due solely to its reduction in laccase activity. Remarkably, after i.n. inoculation of CBA/J mice (Figure 2C), the vps34 strain was rapidly cleared from the lungs, in contrast to WT or 2 previously described hypovirulent knockout strains of the virulence factors laccase $(\operatorname{lac} 1 \Delta)$ or capsule $(\operatorname{cap} 60 \Delta)(18,19)$. Since there was a slight reduction in fungal CFU at the first time point 1 hour after inoculation (WT, $5.8 \pm 0.6 \times 10^{4} \mathrm{CFU} /$ lung vs. $v p s 34 \Delta$, $4.4 \pm 0.5 \times 10^{4} \mathrm{CFU} /$ lung), we conducted a second set of experiments focusing on the $v p s 34 \Delta$ mutant strain to see whether a more prompt recovery from mice and addition of a glucose nutrient (1\% glucose in PBS) could prevent lung clearance of the mutant strain. As shown in Figure 2D, initial recovery of fungal strains at 15 minutes after using an inoculum containing glucose resulted in $v p s 34 \Delta$ fungal burden equal to that of WT (WT, $5.0 \times 10^{4}$ $\pm 0.3 \times 10^{4}$ vs. $v p s \Delta, 5.0 \times 10^{4} \pm 0.4 \times 10^{4} \mathrm{CFU} / \mathrm{lung}$ ) but still showed a rapid clearance compared with WT over the 24-hour time period. In addition, simultaneous inoculation of mice with the vps $34 \Delta$ mutant strain containing an equivalent admixture of WT cells did not protect mutant fungal cells from rapid clearance, suggesting that the protection of WT cells compared with mutants, as shown in Figure 2C, was not due primarily to suppression of clearance mechanisms by the WT cells. (Differentiation of cryptococcal WT from vps $34 \Delta$ mutant cells in mixtures 
A

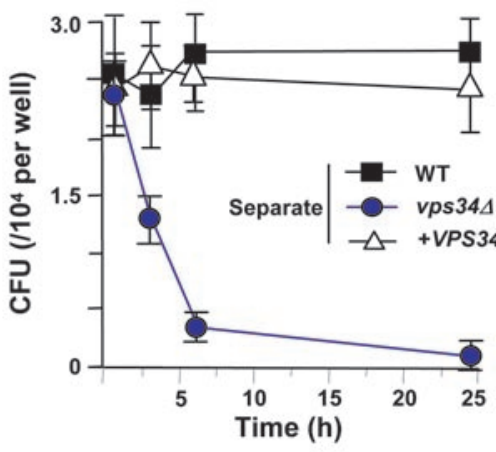

C

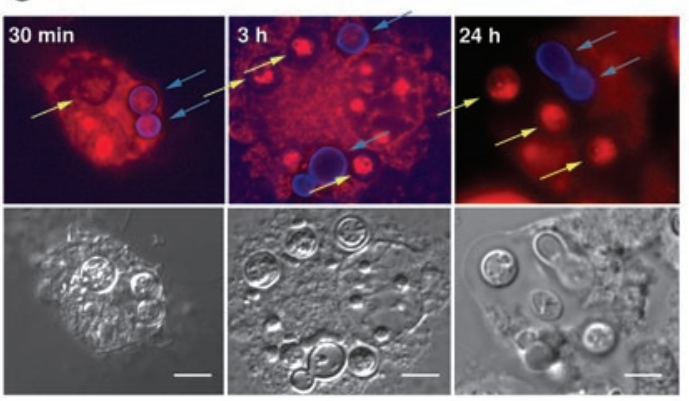

B

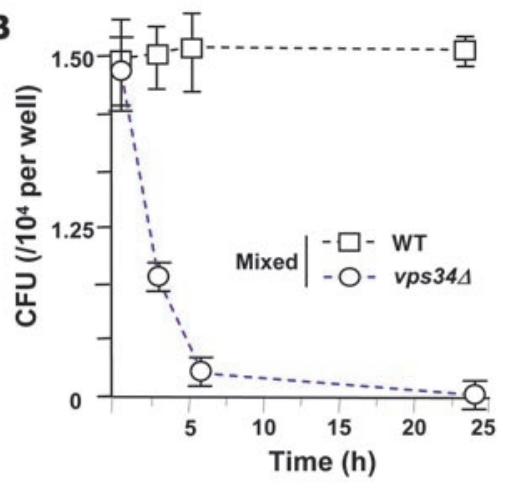

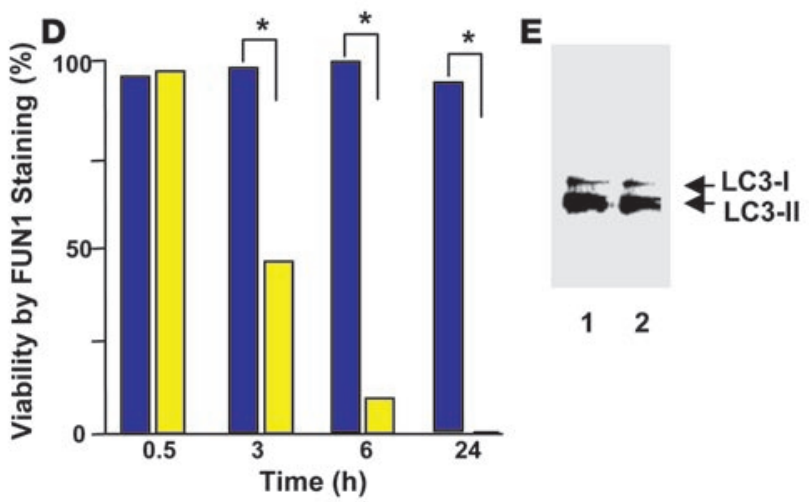

Figure 3

Survival of VPS34 strains in a J774.16 macrophage cell line. (A) Fungal cells were opsonized with anti-capsular antibody, and $10^{5}$ cells were incubated with $\mathrm{J} 774.16$ macrophages for $30 \mathrm{~min}$, washed extensively to remove non-phagocytosed cells, and at the indicated times, wells were washed with water containing $0.01 \%$ SDS and cultured for growth of fungal cells on YPD ( $n=3$ for each strain at each time point.) (B) Same as in A, but macrophages were incubated with $10^{5}$ of a 50:50 mixture of WT and vps34 $\Delta$ mutant cells, and strains were identified from recovered mixtures as described in Methods. (C) Same as in B, but vps34 3 mutant strains were prestained with calcofluor (blue) prior to macrophage inoculation and fungal viability determined by FUN-1 epifluorescence (red) at the indicated times. Blue arrows indicated vps34 $\Delta$ cells, yellow arrows indicate WT cells. Scale bars: 5 microns. (D) Analysis of viable fungal cells in macrophage by FUN-1 staining. Macrophages containing mixtures of WT (unlabeled, blue bars) and vps34 4 mutant (calcofluor-labeled, yellow bars) cells were identified and fungal cells scored by the presence of FUN-1 staining at the indicated times ( $\left.{ }^{*} P<0.001\right)$. (E) Macrophages were infected with either WT or vps $34 \Delta$ mutant fungal cells as in $\mathbf{A}$, and at $3 \mathrm{~h}$, macrophage protein was harvested and subjected to 15\% SDS-PAGE, followed by western blot using 1:1000 anti-LC3 antibody and developed using a horseradish peroxidase anti-rabbit antibody as described in Methods. Lane 1 corresponds to macrophages infected with WT cells and lane 2 to macrophages infected with vps34 3 mutant fungal cells.

recovered from lung was made by laccase assay of 100 cells and confirmed by PCR using specific primers).

In addition, the rapid clearance of the vps34 $\Delta$ mutant cells was accompanied by a vigorous host response as measured by the early procytokine marker TNF- $\alpha$. TNF- $\alpha$ is induced during human and murine cryptococcosis (20) and is normally present 3-7 days after pulmonary inoculation with intact fungal cells (21). In as little as 5 hours, TNF- $\alpha$ production was significantly induced after inocula-

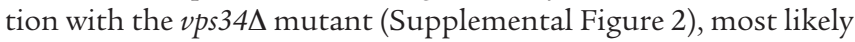
a result of host exposure to liberated fungal proteins from rapidly dying mutant cells, which has been shown previously to induce this cytokine (22). These data show the importance of the VPS34 locus during murine cryptococcosis.

Role of VPS34 and autophagy during macrophage residence of $C n$. Since $\mathrm{Cn}$ is found predominantly within macrophages during lung infection, we examined the survival of the vps34 $\Delta$ mutant strain after phagocytosis by a J774.16 macrophage cell line as previously described (23). As shown in Figure 3A, while WT strains exhibited good survival after macrophage phagocytosis, the vps $34 \Delta$ mutant strain was rapidly killed over a similar time frame as that exhibited in mouse lungs. Control experiments showed that the $v$ ps $34 \Delta$ mutant strain showed greater than $95 \%$ survival in tissue culture medium over the time period tested (data not shown). The clearance of the $v p s 34 \Delta$ strain was also more rapid than mutants of the 2 best-known virulence factors, laccase (lac1 $\Delta$ ) and capsule $($ cap60 4 ) (data not shown). However, the degree of phagocytosis was similar between WT and $v p s 34 \Delta$ cells (WT: $32 \% \pm 8 \%$; $v p s 34 \Delta$ : $40 \% \pm 8 \%$, mean \pm SEM; $P>0.10$ ). In addition, we conducted mixing experiments to determine if the relative survival of the WT mutant fungal strains was due to differential effects on host macrophages. The macrophage cell line was again induced as in experiments shown in Figure 3A but was inoculated with a 50:50 mixture of WT and $v p s 34 \Delta$ cells, followed by removal of non-adherent cells and recovery of fungal cells at the indicated times by lysis of macrophages and quantitation on YPD agar. As shown in Figure $3 \mathrm{~B}$, addition of WT cells to the $v p s 34 \Delta$ cells did not result in protection of mutant cells, suggesting that differences in survival between the strains was not due to suppression of macrophage killing mechanisms by WT cells. In addition, to confirm the mixing studies result, an equivalent macrophage-killing experiment 
A

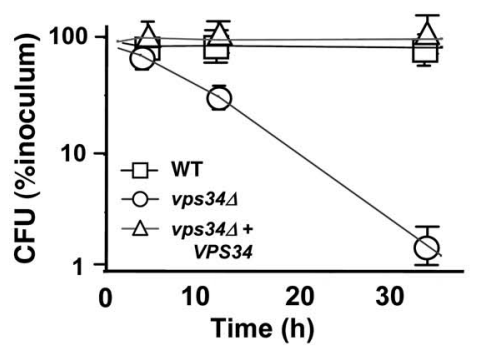

B

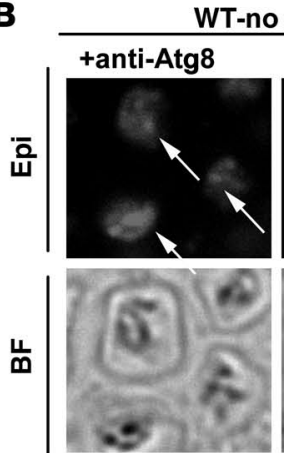

WT-no gluc

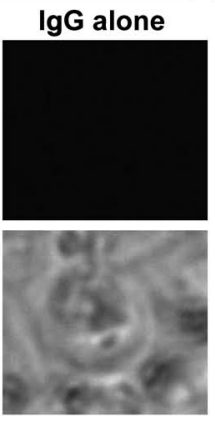

D
WT + gluc +anti-Atg8
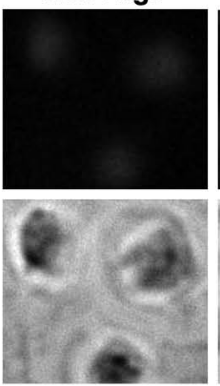

vps344-no gluc +anti-Atg8
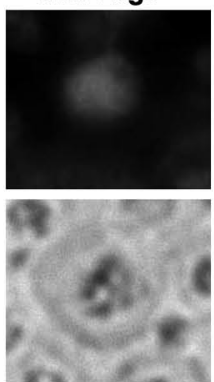

Immunoprecipitation
C

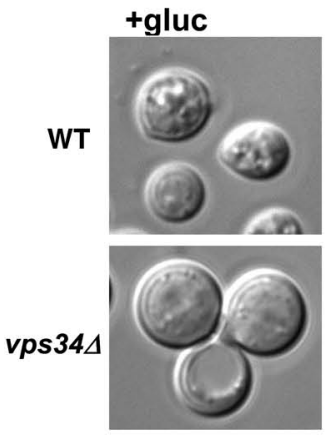

no gluc

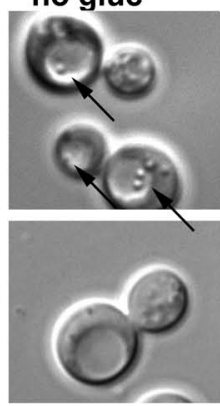

western

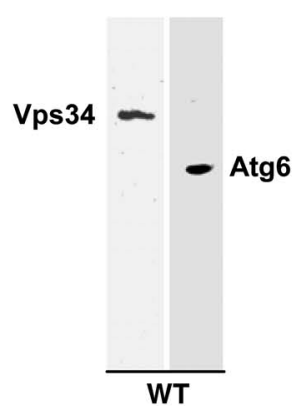

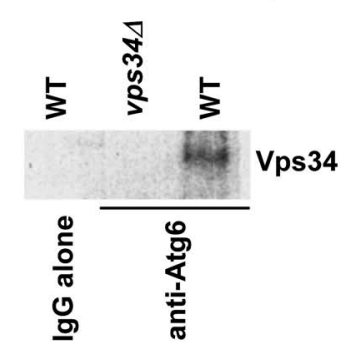

Figure 4

Tolerance of VPS34 strains to nutrient starvation is associated with autophagic processes. (A) Starvation survival: Indicated log phase cells (grown in YPD) were incubated in YNB media without amino acids or ammonium sulfate at $37^{\circ} \mathrm{C}$ for the indicated times, followed by removal of aliquots, and cultured on YPD agar. (B) Indicated log phase cells were harvested (+gluc) or inoculated in YNB without amino acids or ammonium sulfate for $4 \mathrm{~h}$ at $37^{\circ} \mathrm{C}$ (no gluc) and labeled with anti-Atg8 antibody (+anti-Atg8) or rabbit polyclonal lgG (IgG alone), followed by anti-rabbit Alexa Fluor 594, and observed by for epifluorescence (Epi) or bright field (BF). (C) Indicated log phase (+gluc) or log phase cells incubated in YNB without amino acids or ammonium sulfate containing $1 \mathrm{mM} \mathrm{PMSF}$ and $10 \mu \mathrm{g} / \mathrm{ml}$ nocodazole for $4 \mathrm{~h}$ (no gluc) were harvested and subjected to DIC microscopy. Arrows point to autophagic bodies within vacuoles. (D) Indicated cells were subjected to starvation conditions as in C, and whole-cell lysates (left panel) were subjected to western blot using anti-Vps34 antibody or anti-Atg6 antibody or immunoprecipitated with antiAtg6 antibody or rabbit IgG, washed and eluted, and subjected to western blot using the indicated antibodies.

was conducted using a 50:50 mixture of WT and $v p s 34 \Delta$ cells, the latter labeled by a trace amount of calcofluor that was shown not to affect cell viability. After removal of non-adherent cells, macrophage cultures were followed by microscopy, and at the indicated times, macrophages containing both WT and mutant cells were assayed for fungal cell viability by FUN-1 staining, used previously to assess cryptococcal viability in macrophages (24). As shown in Figure 3C, at 30 minutes, both WT and mutant cells showed good viability evident by intact FUN-1. FUN-1 staining was also evident within the cytoplasm of the metabolically active macrophage as previously described during fungal phagocytosis (24). However, over the 24-hour observation period, the vps34 $\Delta$ mutant cells selectively lost viability, whereas the WT cells were unaffected in the same macrophage, similar to that expected based on the CFU mixing studies $(P=\mathrm{NS}$ at $30 \mathrm{~min}, n=45 ; P<0.001$ at $3 \mathrm{~h}, n=54$; $P<0.001$ at $6 \mathrm{~h}, n=42 ; P<0.001$ at $24 \mathrm{~h}, n=53$ fungal cells counted; see Figure 3D). In addition, infection of J774.16 macrophages with either WT or vps $34 \Delta$ mutant cells showed equivalent expression and physiologic cleavage of the mammalian Atg8 homolog LC3-I into LC3-II in the macrophage cell line (Figure 3E), indicative of equivalent induction of macrophage autophagy, which occurs after IF-G-stimulated macrophage killing (25).

Since VPS34 has been implicated in resistance to starvation due to a role in autophagy (8), we next performed survival stud- ies in vitro under starvation conditions. Indeed, in whole-genome microarray experiments, the autophagy-related genes ATG3 and ATG9 were shown to be upregulated in macrophages, although fungal autophagy was not demonstrated (26). As shown in Figure $4 \mathrm{~A}$, the Cn $v p s 34 \Delta$ cells showed poor tolerance to starvation and the rates of fungal cell death were comparable with that exhibited after phagocytosis by J774.16 cells (comparison of slopes of $\log$ survival of $v p s 34 \Delta$ between data in Figure 2D and Figure 3A; $P>0.10)$. In contrast, $v p s 34 \Delta$ mutant cells showed vigorous growth under other conditions expected in macrophages such as oxidative stress (Supplemental Figure 3). Examination of $\nu p s 34 \Delta$ cells under starvation conditions revealed defects in properties attributable to the process of autophagy. For example, while WT cells displayed evidence of autophagy-related (Atg8) association into vesicular structures in response to starvation conditions, the $v p s 34 \Delta$ mutant cells showed a diffuse cytoplasmic localization (Figure 4B). Atg8 is a microtubule-associated protein that is induced and associated with vesicles during autophagy, and this vesicle-associated phenomenon has been used extensively as a marker of autophagy (27). Interestingly, as shown in Figure 4B, Atg8-labeled autophagic bodies were distributed throughout each cell, as opposed to the typical appearance of autophagic bodies principally within large vacuoles in yeast (28), most likely due to formation of smaller multiple vacuolar compartments, which is a characteristic of Cn (29). 
A
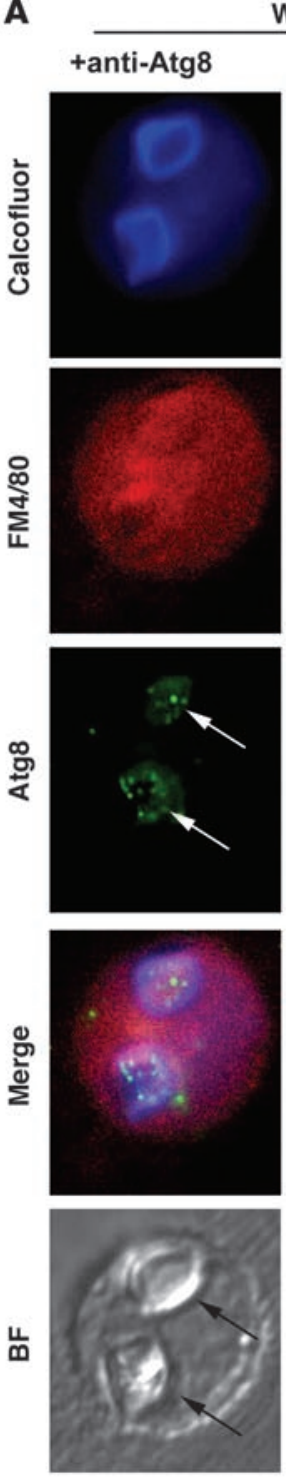

WT
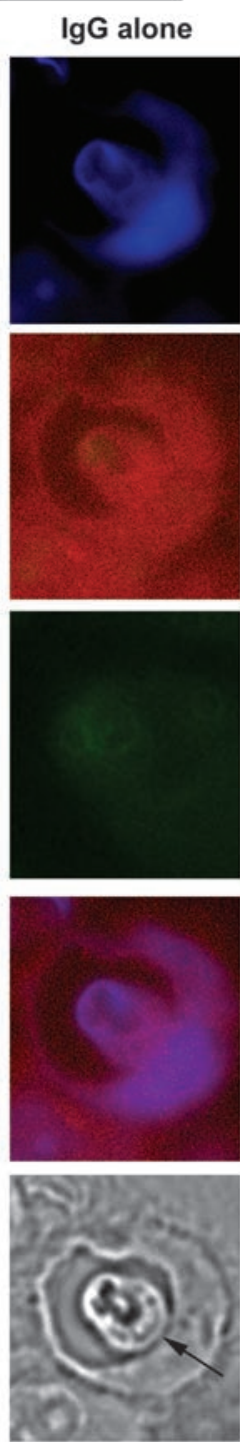
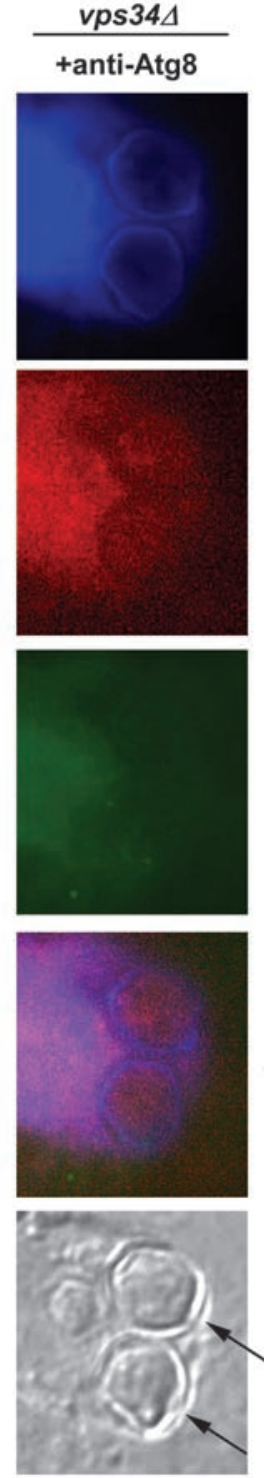

B
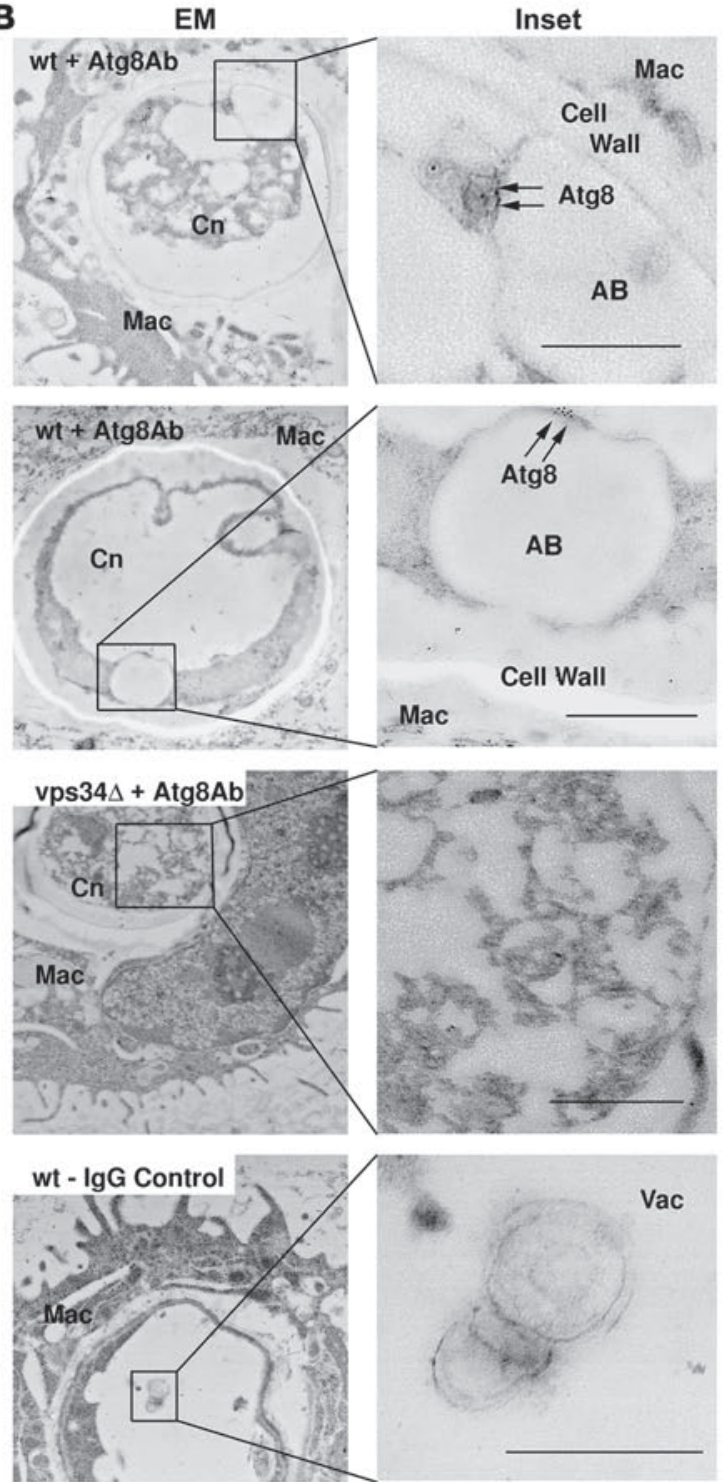

\section{Figure 5}

Formation of autophagic bodies in Cn after macrophage phagocytosis. Indicated cells were subjected to phagocytosis by $\mathrm{J} 774.16$ cells as in Figure 3A, followed by fixation and embedding, and stained with anti-Atg8 antibody (+anti-Atg8) or rabbit IgG (IgG alone) and followed by an anti-rabbit Alexa Fluor 488 fluorescent antibody and anti-FM4/80 Alexa Fluor 594 antibody and observed for epifluorescence (A) or an anti-rabbit 5-nm gold-labeled antibody and observed by electron microscopy (B). AB, autophagic bodies; Mac, mouse macrophage; Vac, vacuole. Arrows point to intracellular fungal cells in A and gold particle labeling of Atg8 in B. Scale bars: $500 \mathrm{~nm}$.

However, addition of the vacuolar-modifying microtubular inhibitor nocodazole (30) resulted in the formation of larger vacuoles in Cn during starvation conditions, which allowed better visualization of motile autophagic bodies using DIC microscopy under starvation conditions. Using this protocol, autophagic bodies were observed within WT cells but not in identically treated vps34A mutant cells (Figure 4C and Supplemental Movie). Analysis of multiple cryptococcal cells revealed a number of autophagic bodies in WT cells (range, $0-5$; mean \pm SEM, $2.0 \pm 0.4 ; n=15$ ), whereas none were observed in vps34 cells $(n=15 ; P<0.001)$. Furthermore, because of the conserved nature of a number of autophagic proteins including Vps34 described above, Atg6 (22\% similarity between Sc and Cn) and Atg8 (89\% similarity between Sc and Cn) rabbit antibodies against Sc Vps34, Sc Atg6, and Sc Atg8 cells were tested and showed immunoreactive bands equal to their predicted sizes $(101,63$, and $14.5 \mathrm{kDa}$, respectively), with cryptococcal extracts on western blot (Figure 4D and Supplemental Figure 4). These immuno-crossreactive antibodies were used to show formation of autophagic complexes during starvation, demonstrated by coimmunoprecipitation of Vps34 by the autophagic protein Atg6, as reported previously in yeast $(9,31)$. Recruitment of autophagyrelated proteins by Vps34 is an important step in the formation of the autophagic complex in yeast, and these data support a similar mechanism in $\mathrm{Cn}$.

To determine whether autophagic processes occurred during macrophage residence of cryptococcal cells, vesicularization of 
A

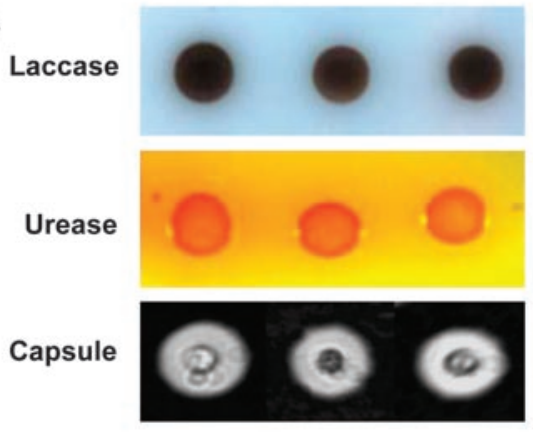

Control 1 Control 2 IATG8

C

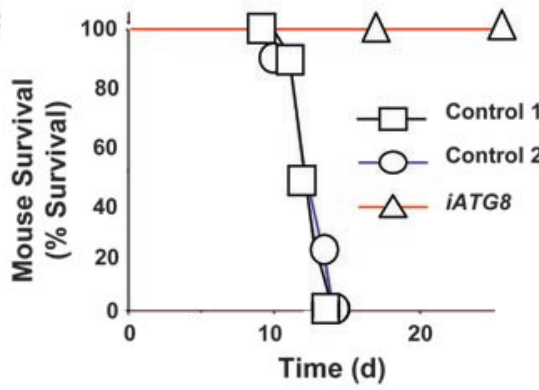

E

+anti-Atg8

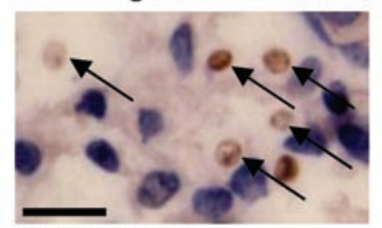

mucicarmine

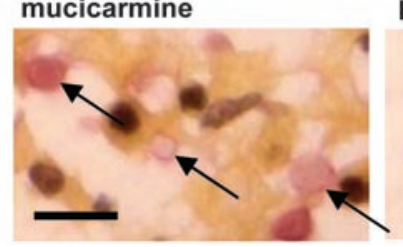
lgG control
B
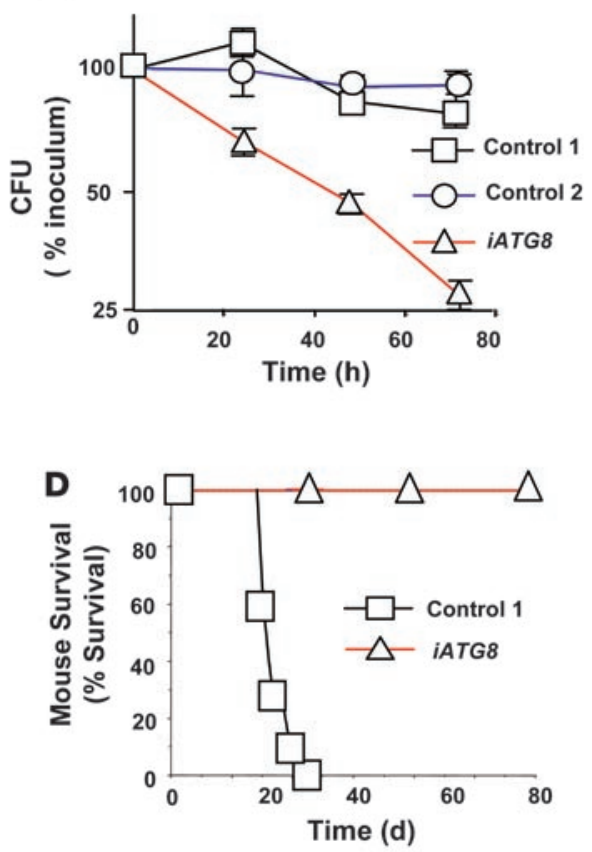

F

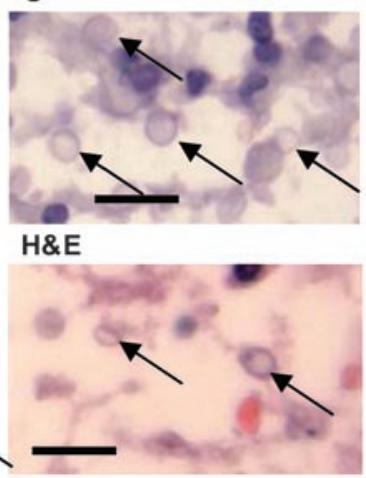
+anti-Atg8

IgG control

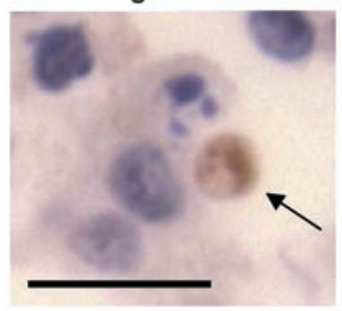

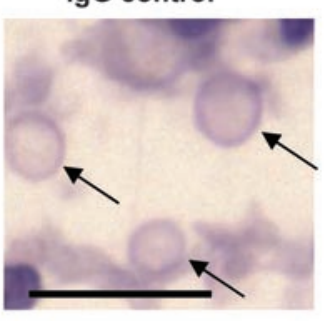

Figure 6

Role of ATG8 in murine and human cryptococcosis. (A) Cn cells transformed with the ATG8 RNAi construct (iATG8) or control plasmid (controls 1 and 2) assayed for laccase, urease, and capsule, as described in Methods. (B) Indicated mid-log phase strains incubated in YNB without amino acids or ammonium sulfate for the indicated times and cultured for growth of fungal cells on YPD. (C) Indicated strains $\left(10^{6} \mathrm{CFU}\right)$ were inoculated intravenously into Swiss Albino mice and followed until moribund. (D) Indicated strains ( $10^{5} \mathrm{CFU}$ ) were inoculated intranasally into CBA/J mice and followed until moribund. (E) Antibody staining of Cn-infected human brain tissue incubated with an anti-Atg8 antibody (+anti-Atg8) or rabbit IgG (IgG control) as well as an anti-rabbit horseradish peroxidase secondary antibody and developed with diaminobenzidine and counterstained with H\&E. Lower panels show mucicarmine and H\&E stain of equivalent tissue. (F) Higher magnification of intracellular fungal cells stained as in E. Arrows point to fungal cells. Scale bars: $10 \mu \mathrm{m}$.

Atg8 was investigated after phagocytosis by the J774.16 macrophage cell line. Cryptococcal cells were identified by cell wall staining by the dye calcofluor, which also demonstrated some fluorescence with macrophages, as previously described (32). As shown in Figure 5A, J774.16 phagocytosis of WT but not vps $34 \Delta$ mutant cells resulted in formation of Atg8-immunoreactive vesicles. This was visualized by epifluorescence after 3 hours of phagocytosis by the FM4/80-stained macrophage cell line and by the formation of Atg8-stained fungal autophagic bodies visualized by immunoelectron microscopy (Figure 5B). Most likely, induction of autophagy in phagocytosed fungal cells is the result of exposure to a nutrient- poor phagolysosome in the macrophage, suggested recently by a requirement for virulence of phosphoenolpyruvate carboxykinase, which is a gluconeogenic enzyme required to synthesize glucose under carbon starvation conditions (5).

Reduced tolerance to starvation and virulence of an iATG8 knockdown strain. To molecularly dissect the contribution of autophagy to the VPS34 phenotype during cryptococcal infection, a reduction in autophagic capacity was produced by RNA interference of the autophagy-related gene ATG8. Control strains were produced by transformation of fungal cells with an equivalent plasmid in equivalent copy number but not containing the RNAi construct. 
The Cn iATG8 strain retained WT growth under nutrient-replete conditions at $37^{\circ} \mathrm{C}$ (see Methods) and retained the virulence factors laccase, urease, and capsule (Figure 6A). However, the iATG8 strain showed sensitivity to starvation (Figure $6 \mathrm{~B}$ ), although not quite as severe as the $v p s 34 \Delta$ strain, which could be due to residual ATG8 expression in the iATG8 knockdown strain (see Supplemental Figure 4B). The iATG8 knockdown strain also showed attenuated virulence in the intravenous mouse model (Figure 6C) as well as in an intranasal model (Figure 6D) compared with the control strains, with only small amounts of residual iATG8 organisms noted in brains of sacrificed animals $\left(<10^{3} \mathrm{CFU} /\right.$ brain $)$. To determine whether cryptococcal Atg8 is expressed during human CNS disease, Atg8 immunoreactivity was demonstrated in fungal cells during infection of human brain (Figure 6, E and F), which showed vigorous Atg8 expression in fungal cells. In contrast, we did not see significant Atg8 reactive signal throughout brain tissue or on western blots of mouse brain (Supplemental Figure 4), suggesting that the Atg8 antibody was yeast specific. In summary, these studies demonstrate the role of VPS34 signaling and autophagy in starvation response and in the pathogenesis of cryptococcosis.

\section{Discussion}

In the present study, identification and deletion of a VPS34 gene associated with the virulence factor laccase allowed for the analysis of the role of inositol phosphate signaling, autophagy, and starvation response in the pathogen $\mathrm{Cn}$. This expands further the genetic relationships between an immune modulatory virulence factor, laccase $(33,34)$, and starvation response programs such as autophagy that play a central role during virulence. Previously, regulation of laccase activity has been genetically linked to processes important under nutrient-limiting conditions such as gluconeogenesis (5) and high-affinity copper acquisition (6). Such a genetic association between laccase and attributes such as autophagy, important to survival during the search for nutrients, recalls the organism's environmental niche within the hollows of trees where laccase is involved in the generation of energy by the use of alternative substrates including degradation of wood lignins in the absence of glucose (35). The existence of such strong genetic relationships between virulence determinants and environmental genetic programs are a testament to the success of opportunistic pathogens such as $\mathrm{Cn}$ that appear to effortlessly switch between an environmental saprophyte and a virulent pathogen. In addition, laccase's extensive relationships to virulence determinants provides additional insights into why laccase and its product melanin has proven successful as a strong marker of pathogenic strains of Cryptococcus (36).

Autophagy is an evolutionarily conserved response to stress and has been linked to important protective roles in mammalian cells such as lifespan extension and protection from diverse human diseases including cancer, muscular disorders, neurodegeneration (e.g., Huntington, Alzheimer, and Parkinson diseases) (37-39). In the mammalian host-pathogen interaction, the role of autophagy has mostly favored the host, as induction of autophagy in macrophages has been found to be key in the control of replication of diverse prokaryotes such as Mycobacterium tuberculosis and Legionella pneumophila within autophagic vesicles $(25,40)$. However, the present studies indicate that PI3K-dependent induction of autophagy is also a tool used by the eukaryotic pathogen to ensure survival and effect host cell damage. Interestingly, the fungal pathogen Candida albicans has not been found to be dependent on autophagy for virulence (41), although a reduction in virulence was found by deletion of a $v p s 34 \Delta$ homolog (42), which may suggest that the 2 pathogens encounter different host-cell environments leading to varied requirements for an autophagic response. Alternatively, this could be a reflection of pathogen-specific alterations in the host cell environment. For example, Cn liberates an extensive polysaccharide capsule within macrophages which has macrophage modulating properties that could alter the environment of the phagolysosome (43). In addition, Candida species may elicit specific protective properties that improve nutrient conditions similar to Mycobacterium within the host environment (44), making it less dependent on its own autophagosomal machinery. Regardless of mechanisms, these different requirements for autophagy between pathogens offer insights into the unique aspects of interactions between fungi of different species and the mammalian host.

Induction of autophagy in Cn was found to be dependent on the PI3K locus VPS34, as previously described in ascomycetous yeast (8). Interestingly, the almost WT growth of the cryptococcal vps $34 \Delta$ mutant at $37^{\circ} \mathrm{C}$ distinguishes it from the temperature sensitivity of the homologous mutation in ascomycetous yeast and may reflect the evolution of higher eukaryotes. However, in spite of robust growth at $37^{\circ} \mathrm{C}$, the cryptococcal vps $34 \Delta$ mutant showed a remarkably fast clearance from lungs of mice, which was associated with a defect in tolerance of starvation and formation of autophagic bodies. Such extremely different survival patterns of the vps $34 \Delta$ mutant in tissue culture media containing glucose compared with survival patterns in the host environment suggest that the former is not representative of host conditions. This discrepancy in pathogen response between these 2 conditions may have implications for the selection of conditions used to mimic the host environment, such as the determination of antifungal minimum inhibitory concentrations (MICs). These analyses, used to predict antifungal resistance during clinical infections, were conducted under nutrient-replete conditions as recommended by the National Committee for Clinical Laboratory Standards $(45,46)$. Unfortunately, cryptococcal fungal MICs have shown a remarkable inability to predict clinical endpoints (47), which could be partly due to differences in antifungal resistance within differing nutrient environments.

In summary, while these data suggest that an effective response, such as PI3K signaling and autophagy, by the pathogen to starvation encountered during infection may be detrimental to the host, they also suggest how attention to fungal stress response pathways may lead to more effective preventative or treatment modalities tailored to the unique environment of the host.

\section{Methods}

\section{Fungal strains, plasmids, media, and genomic databases}

Cn ATCC 208821 (H99) was a generous gift of J. Perfect (Duke University, Durham, North Carolina, USA). Strain H99FOA19 (H99 Mata, ura5) (29) was employed as a recipient strain for creating targeted gene deletion. For starvation experiments, the strains were grown in YPD media ( $2 \%$ glucose, $1 \%$ yeast extract, $2 \%$ Bacto-peptone) or $2 \%$ glucose in yeast nitrogen base (YNB; Becton Dickinson) or were incubated in YNB without amino acids or ammonium sulfate (Becton Dickinson) media. Asparagine minimum selective media for transformant selection and for detection of laccase production were previously described (48). Plasmid PPM8 and pCIP containing the URA5 gene was a kind gift of K.J. Kwon-Chung (NIH, Bethesda, Maryland, USA). Plasmids containing the hygromycin resistance gene was a gift of G. Cox (Duke University, Durham, North Carolina, USA). 
Sc WT (BY4730) and vps34 mutant strain clone ID 15149 was acquired from Opensystems Inc. Cryptococcal genes were identified using the serotype D TIGR genomic database (http://www.tigr.org/tdb/e2k1/cna1) as well as the Cn serotype A (http://www.broad.mit.edu) and UniProt (http:// beta.uniprot.org) databases. Clustal-W analysis was determined as previously described (14) using the EMBL-EBI database (http://www.ebi.ac.uk/ Tools/clustalw/index.html).

\section{Cell lines and culture media}

J774.16 (ATCC) is a murine macrophage-like cell line derived from a reticulum sarcoma. Cells were maintained at $37^{\circ} \mathrm{C}$ in $5 \% \mathrm{CO}_{2}$ in DMEM (Invitrogen) supplemented with 10\% heat-inactivated FCS (Harlan Sprague Dawley Inc.), Cellgrow $1 \times$ nonessential amino acids (Mediatech), penicillin/streptomycin, and 10\% NCTC-109 medium (Invitrogen). Cell lines were used between 5 and 15 passages.

\section{Disruption and complementation of VPS34 in Cn and in Sc}

Standard methods were used for disruption and complementation of the VPS34 gene, as described previously (29). Briefly, to make the deletion construct, 2 PCR-amplified fragments of the Cn VPS34 homolog (using primers VPS34-14446S-XbaI, 5'-GCCGGCTCTAGAGTGTGTCTTCCTCGACA, and VPS34-1989A-RI, 5'-CCACGAATTCTGTGGAGGGTGAGAGTTG; VPS34-4656S-BgII, GCCGCCAGATCTGCCCATTATGGAAGATC, and VPS34-5055A-XhoI, 5'-GCCGCCCTCGAGTATGGGTAGTTATACATCTTG), the first digested with $X b a \mathrm{I}$ and $E c o R \mathrm{I}$ and the second digested with $B g l I$ and $X h o I$, was mixed with a $1.3-\mathrm{kb}$ PCR fragment of the Cn URA5 gene described previously (49), digested with BglII and EcoRI and ligated to BlueScript SK digested with $X b a I$ and $X b o I$. The final disruption allele with a 1.3-kb URA5 marker flanked on either side by a 500-bp DNA sequence homologous to genomic regions of the VPS34 gene was PCR-amplified and introduced into H99FOA cells via a biolistic approach (50) to effect a $2.6-\mathrm{kb}$ deletion within the VPS34 coding region. Transformants were screened for potential VPS34 deletion mutant by a PCR approach, and the specific disruption of the VPS34 gene in candidate mutants was verified by Southern blot analysis (Supplemental Figure 1). To complement the vps34 $\Delta$ mutant strain, a 6.3-kb genomic fragment encompassing the full ORF of VPs 34 plus $\sim 1200$ bp of the $5^{\prime}$ promoter region was PCR amplified using a primer set of VPS34-5-S-ClaI (5'-CCATCGATGGTTCTACCTCTTCACTCTCAGA-3') and VPS34-6569-A-ClaI (5'-CCATCGATGGGATCGTCCACTACCGAATTAT- $3^{\prime}$ ) and then cloned into a modified Bluescript SK vector (Stratagene) containing the $2-\mathrm{kb}$ hygromycin- $\mathrm{B}$ resistance gene under the control of a cryptococcal actin promoter (50) to generate the complementation construct, which was introduced into $v p s 34 \Delta$ mutant cells by electroporation, as previously described (29), and transformants were selected on hygromycin-containing YEPD agar plates $(150 \mathrm{U} / \mathrm{ml})$. Heterologous genomic insertion of the WT VPS34 and retention of the URA5 deletion construct in the complement was verified by uncut Southern blot and PCR. Complementation of a Sc mutant with Cn VPS34 was effected by overexpression of a PCR-amplified copy of VPS34 from a previously described Cn H99 cDNA library (29) in the Sc CEN expression vector pYX-112 (R\&D Systems; a gift of D. Askew, University of Cincinnati, Cincinnati, Ohio, USA) under the TPI promoter using primers vps34-2076-S-AvrII (5'-GGCCCTAGGATGAGTCTGTTCAACTCTAAGC-3') and vps34-4319-A-XhoI (5'-CCGCTCGAGCTAATCCCGAAGATACTGCG-3') after endonuclease digestion with AvrII and $\mathrm{XhoI}$ and ligation into compatible sites of the PYX-112 yeast vector followed by recovery, sequencing, and transformation into the Sc vps34 mutant strains. Control transformations were effected by transformation with the PYX-112 empty vector alone.

PI3K activity was determined by the method of Storz et al. (51). Briefly, cell extract of indicated strains and PI3K activity assayed by addition of
$10 \mu \mathrm{g}$ sonicated phosphatidylinositol in $30 \mathrm{mM}$ Hepes buffer, $\mathrm{pH}$ 7.4, $30 \mathrm{mM} \mathrm{MgCl}_{2}, 200 \mu \mathrm{M}$ adenosine, $50 \mu \mathrm{M}$ ATP, $20 \mu \mathrm{Ci}\left[\gamma^{-32} \mathrm{P}\right] \mathrm{ATP} \pm 0-50$ $\mathrm{nM}$ wortmannin for $10 \mathrm{~min}$, followed by analysis of phosphate products by thin-layer chromatography by the buffer system of Storz et al. as well as Chen (15), with reference to standard PI3P and PI4P (Sigma-Aldrich).

\section{RNA interference}

RNAi suppression of ATG 8 was performed by a method similar to that described previously (5). The ATG8 gene was PCR amplified from an H99 cDNA mass-excised library using primers ATG8-2238S-RI (5'-GCCGCGAATTCTGTGAGAAGGCTGAGAAGA) and ATG8-2859A-XhoI (5'-GCCGCCCTCGAGTTAAACATAGTTGCTTTGCTTA) and 2 fragments of the $A T G 8$ gene were ligated downstream from an $A C T 1$ promoter of a PORA-KUTAP cryptococcal shuttle vector described previously (17), in a sense-antisense fashion on either side of a linker fragment consisting of a 500-bp PCR-amplified fragment of intron I of LAC1 generated from a $L A C 1$ genomic clone using primers INTRON-XhoS and INTRONXhoA to produce plasmid ORA-i-ATG8. ORA-i-ATG8 was linearized and transformed into H99 Mata, ura5-19 described previously (29), and transformants selected on minimal media. Control strains (controls 1 and 2) were produced by transforming a H99 Mat $\alpha$ ura5 strain with an identical pORA-KUTAP plasmid without the ATG8 RNAi construct. While URA transformation markers have been problematic for virulence studies in some fungi such as Candida due to gene dosing of the inserted uracil marker (52), Cn virulence does not appear to be as sensitive to levels of uracil synthesis and, for example, all single loci disruptions using URA5 in this fungus from our laboratory have been successfully complemented with full restoration of virulence in strains having heterologously localized URA5 genes (53). Nevertheless, to control for URA5 expression between strains, 2 independent transformants (controls 1 and 2) carrying control pORA-KUTAP plasmids and 1 containing the same plasmid with the above iATG8 RNA interference construct were selected based on equivalent copy number determined on uncut Southern blots as described and were matched within $20 \%$ for equivalent expression of URA5 (ratio of URA5 to rRNA by densitometry) by northern blot analysis (see Supplemental Figure 4). Growth of the 3 strains was equivalent as measured by doubling time in $2 \%$ glucose, $\mathrm{YNB}$ at $37^{\circ} \mathrm{C}$ (control 1, $3.1 \pm 0.4$; control 2, $2.8 \pm 0.4$; iATG8, $3.0 \pm 0.2 \mathrm{~h}$, mean $\pm \mathrm{SEM}$ ). In addition, in control experiments testing for the ability of strains expressing the control KUTAP plasmids to produce virulence in mice, the WT H99, control 1, and control 2 strains were injected intravenously into 10 mice per group (inoculum, $1 \times 10^{6} \mathrm{CFU}$ ) and followed until moribund, demonstrating that survival times were not significantly different between WT $\mathrm{Cn}$ (H99) and the 2 strains containing the KUTAP plasmid (mean time of death \pm SEM: H99 WT, $11 \pm 0.5 \mathrm{~d}$; control 1 , $12.6 \pm 0.5 \mathrm{~d}$; control 2, $12.5 \pm 0.4 \mathrm{~d}, P>0.05$ by ANOVA between all groups).

\section{Macrophage killing assays}

The J774.16 macrophage-like cell line (ATCC) was used to evaluate the ability of the $v p s 34 \Delta$ mutant strains to grow inside macrophages by a previously described method (54). Cells were allowed to grow for 5-7 days in DMEM (Cellgro) supplemented $10 \%$ fetal calf serum, $100 \mu \mathrm{g} / \mathrm{ml}$ Cellgro penicillinstreptomycin at $37^{\circ} \mathrm{C}$ in the presence of $5 \% \mathrm{CO}_{2}$, and then harvested from monolayers using $0.25 \%$ trypsin, and the number of cells were counted with a hematocytometer. The macrophage concentration was adjusted to $10^{6} \mathrm{cells} /$ $\mathrm{ml}$, and $100 \mu \mathrm{l}$ of the macrophage suspension was added to each well of a 12 -well plate. The cells were primed with murine IFN- $\gamma$ (Sigma-Aldrich) at a concentration of $50 \mathrm{U} / \mathrm{ml}$ and were incubated at $37^{\circ} \mathrm{C}, 5 \% \mathrm{CO}_{2}$ overnight. Yeast cell suspensions $\left(10^{7} / \mathrm{ml}\right)$ of indicated fungal cells were prepared from fresh cultures and antibody 18B7 (IgG1) (a generous gift of A. Casadevall) added $(10 \mu \mathrm{g} / \mathrm{ml})$ and incubated at $37^{\circ} \mathrm{C}$ for $30 \mathrm{~min}$. Viability of the inocu- 
lum was determined to be $>80 \%$ by CFU measurement in each case. To each well in the 96-well plate, $10^{6}$ antibody-treated cryptococcal cells were added plus 50 units of IFN- $\gamma$ (Sigma-Aldrich) and $1 \mu \mathrm{g}$ LPS and incubated at $37^{\circ} \mathrm{C}$ $5 \% \mathrm{CO}_{2}$. The macrophage and yeast mixtures were incubated for $30 \mathrm{~min}$, and extracellular yeast cells were removed by washing with PBS 3 times. Parallel experiments using the macrophage impermeant dye uvitex $2 \mathrm{~B}$ showed that greater than $90 \%$ of adherent cells were intracellular by the method of Levitz (55). At the indicated times after addition, macrophages were lysed with $0.1 \%$ SDS in water. Pilot experiments showed that incubation in $0.1 \%$ SDS resulted in no loss of viability of $\mathrm{Cn}$ strains. The collected yeast cells were finally washed with and suspended in PBS and plated on chloramphenicol-containing YPD agar for colony counts. For macrophage western blots (Figure 3E), lysis was conducted in the presence of a protease inhibitor cocktail (P-8215; Sigma-Aldrich) and protein separated from intact fungal cells by centrifugation prior to SDS-PAGE. For mixing studies (Figure 3A), 100 recovered fungal cells were assayed for each time point for laccase activity, and WT cells were identified by intact laccase activity. This result was confirmed by PCR amplification of 1 of every 10 strains using specific primers. All experiments were done in triplicate. Macrophage mixing studies were also performed by labeling fungal cells with $17 \mu \mathrm{g} / \mathrm{ml}$ of calcofluor dye, followed by washing to remove excess dye prior to mixing with unlabeled cells and addition to macrophage cultures. Pilot studies showed no loss of fungal viability with calcofluor staining under these conditions. Fungal viability was determined by FUN-1 epifluorescence in macrophages containing both WT and mutant strains identified by calcofluor epifluorescence at the indicated times, as described previously (24). Phagocytic index was calculated as the number of ingested yeast cells per 100 macrophages according to Kozel et al. (56). Phagocytic index was calculated as the mean number of ingested yeast cells per $100 \mathrm{~J} 774.16$ cells \pm SEM; $n=8$ for each group.

\section{Starvation stress response and growth assays}

To assess the tolerance of fungal cells to starvation conditions, yeast cells of overnight cultures in YPD liquid medium of indicated strains were collected, washed, and resuspended to a concentration of $10^{8}$ cells $/ \mathrm{ml}$ in $15 \mathrm{ml}$ YNB without amino acids or ammonium sulfate (Difco) in a 50-ml tube and then cultivated at $37^{\circ} \mathrm{C}$ with shaking at $250 \mathrm{rpm} / \mathrm{min}$. At the indicated time point, $100 \mu \mathrm{l}$ of each strain suspension was removed and used to determine cell viability based on CFU counting on YPD agar using at least a 10,000-fold dilution followed by appropriate serial dilutions. Fungal growth was determined by spotting 1:10 serial dilutions of mid-log phase suspensions of indicated fungal cells on the indicated agar, and quantitative growth curves were performed by incubating log phase fungal cells in the indicated liquid media to a concentration of $1 \times 10^{6} / \mathrm{ml}$ and following serial cell counts at hourly intervals according to the methods of Panepinto (5).

Detection of vacuolar autophagic bodies was based on a modification of the method of Kirisako et al. (28). Previous studies had shown that under starvation response, cryptococcal cells typically form multiple small vacuoles, rather than one large vacuole typical of ascomycete yeast (29), making observation of motile autophagic bodies within these structures difficult. Pilot studies determined that addition of nocodazole, which has previously been shown to modify vacuolar structures (30) but not autophagy (28), resulted in successful visualization of multiple motile vesicles in $\mathrm{Cn}$. Thus, indicated mid-log phase cells were incubated in YNB without amino acids or ammonium sulfate for $4 \mathrm{~h}$ at $30^{\circ} \mathrm{C}$ in the presence of $5 \mathrm{mM}$ PMSF and $10 \mu \mathrm{g} / \mathrm{ml}$ nocodazole and observed by DIC microscopy. Autophagy was also quantitated by the method of Noda et al. (11) by the production of alkaline phosphatase.

\section{Electron microscopy}

The method of Zhu et al. was used for immunoelectron microscopy (57). Briefly, cells were incubated $3 \mathrm{~h}$ after phagocytosis by J774.1 cells, removed by gentle agitation, and fixed in $4 \%$ (vol/vol) paraformaldehyde, $0.05 \%$ (vol/ vol) glutaraldehyde in $100 \mathrm{mM}$ sodium phosphate buffer, $\mathrm{pH} 7.2$ for $16 \mathrm{~h}$ at $4^{\circ} \mathrm{C}$. Cells were then washed 3 times for $10 \mathrm{~min}$ per wash in phosphate buffer, then dehydrated in a graded series of ethanol concentrations $(15 \%$, $30 \%, 50 \%, 75 \%$, and $100 \% \mathrm{vol} / \mathrm{vol}$ ) with 2 changes each for $45 \mathrm{~min}$. Infiltration was continued with 2 parts ethanol to 1 part LR White resin, then 1:1, and 1:2 each for 2 days. Pure LR white infiltration was completed over $24 \mathrm{~h}$ with 3 changes, and the samples were then polymerized in $1 \mathrm{ml}$ of pure LR White resin in a vacuum oven at $50^{\circ} \mathrm{C}$ for $3 \mathrm{~d}$. Cured blocks were trimmed and thin-sectioned with a diamond knife on a Riechert Ultra Cut E ultramicrotome (Leica) and the sections picked up on 200-hex-mesh $\mathrm{Ni}$ grids. The thin sections on $\mathrm{Ni}$ grids were incubated at room temperature in blocking solution $(0.8 \%$ [wt/vol] BSA, $0.1 \%$ [wt $/ \mathrm{vol}]$ immunogold-silver stain-quality gelatin, $5 \%[\mathrm{wt} / \mathrm{vol}]$ normal goat serum in PBS, $\mathrm{pH} 7.4)$ for $45 \mathrm{~min}$, washed twice in $0.8 \%$ (wt/vol) BSA, $0.1 \%$ (wt $/ \mathrm{vol}$ ) gelatin, $0.025 \%$ (vol/vol) Tween 20 in $\mathrm{pH} 7.4 \mathrm{PBS}$, and incubated overnight with 1:200 of a rabbit anti-Atg8 antibody (1:500 dilution) in $0.8 \%$ (wt/vol) BSA, $0.1 \%$ $(\mathrm{wt} / \mathrm{vol})$ gelatin, $1 \%(\mathrm{vol} / \mathrm{vol})$ normal goat serum in BPS, washed twice in washing solution, and incubated with immunogold-labeled secondary antibody (goat anti-rabbit IgG; Amersham) diluted 1:25 for $4 \mathrm{~h}$. Grids were washed 6 times with the washing solution for $5 \mathrm{~min}$ per wash, then twice with PBS, and the samples fixed with $2 \%$ (vol/vol) glutaraldehyde in PBS for 10 min. Grids were then washed twice in PBS and 3 times in distilled water, dried, stained with $2 \%(\mathrm{wt} / \mathrm{vol}$ ) aqueous uranyl acetate for $5 \mathrm{~min}$, dried, stained with lead citrate for 2 minutes, and photographed with a JEOL 1200 EX transmission electron microscope.

\section{Antibody staining, western blotting, and coimmunoprecipitation}

Immunofluorescence of intact cells. The method of Tucker and Casadevall (54) was adapted as follows: Cells were grown to mid-log phase and either harvested or washed twice in sterile water and incubated for the indicated time in YNB without amino acids or ammonium sulfate at $37^{\circ} \mathrm{C}$. Cells were fixed in $3 \%$ formaldehyde for $1 \mathrm{~h}$ at $4^{\circ} \mathrm{C}$, washed extensively, and then subjected to spheroplasting using $40 \mathrm{mg} / \mathrm{ml}$ of lysing enzyme from Trichoderma harzianum (Amersham) in $1 \mathrm{M}$ sorbitol, $10 \mathrm{mM}$ sodium citrate, $\mathrm{pH}$ 5.8, as previously described (58) for $4 \mathrm{~h}$ at $30^{\circ} \mathrm{C}$. Cells were then washed in $1 \mathrm{M}$ sorbitol and $10 \mathrm{mM}$ sodium citrate buffer, diluted 1:8 in PBS, dried on microscope slides, fixed in $100 \%$ anhydrous methanol at $-60^{\circ} \mathrm{C}$, and then dried again. To quench autofluorescence, cells were incubated in $0.1 \mathrm{M}$ glycine for $10 \mathrm{~min}$. Cells were then incubated with a solution of 1:500 dilution of rabbit anti-yeast Atg8 (Abcam) or IgG purified from rabbit serum (Sigma-Aldrich) in PBS containing $1 \mathrm{mg} / \mathrm{ml} \mathrm{BSA}$ (Sigma-Aldrich) at $4^{\circ} \mathrm{C}$ for $1 \mathrm{~h}$, followed by extensive washing in PBS, then incubation for $1 \mathrm{~h}$ at $4^{\circ} \mathrm{C}$ with 1:1000 Alexa Fluor 594 chicken anti-rabbit antibody followed by 3 washes with PBS and immunofluorescence examined using an Olympus IX-70 microscope with Slide Book 4 deconvolution software (Intelligent Imaging Innovations Inc.).

Western blots. Western blots using cryptococcal cell extract, the anti-Atg8 antibody described above (1:5000), rabbit anti-Vps34 (1:2000) (8), or rabbit anti-rat LC3 (1:1000; MBL Medical and Biological Laboratories; ref. 59) and a mouse anti-rabbit HRP antibody (Sigma-Aldrich) $(1: 10,000)$ were performed as previously described (57).

Antibody staining of sectioned yeast cells. WT or vps $34 \Delta$ mutant cells were grown to log phase in YPD, recovered by centrifugation, and processed as for immunoelectron microscopy, except that sections were adhered to glass plates and incubated with a 1:500 dilution of the anti-Atg8 antibody or purified rabbit polyclonal IgG as described above followed by a 1:500 HRP-labeled anti-mouse antibody.

Coimmunoprecipitation. Coimmunoprecipitation was performed by an adaptation of the method of Zhang et al. (60). Briefly, rabbit anti-Atg6 
or purified rabbit polyclonal IgG was linked to G-Sepharose and cross linked using the Seize X Protein G immunoprecipitation kit according to the manufacturer's directions (Pierce Biotechnology). Indicated mid-log phase $\mathrm{Cn}$ cells were incubated in YNB without amino acids or ammonium sulfate for $3 \mathrm{~h}$ at $30^{\circ} \mathrm{C}$, and cell lysate $(1 \mathrm{mg})$ was incubated with the linked Sepharose, followed by extensive washing, elution, and western blotting using the indicated antibodies as described above.

\section{Cytokine analysis}

Cytokine analysis was conducted as previously described (61). Briefly, lungs were excised and removed on ice under aseptic conditions, rinsed 3 times in PBS, weighed, and rinsed in antibiotic-containing $(100 \mathrm{U} / \mathrm{ml}$ penicillin, $100 \mathrm{mg} / \mathrm{ml}$ streptomycin) RPMI-1640 (Sigma-Aldrich), and 1 lung of each mouse was homogenized on ice and the other was subjected to fixation and histologic preparation. The collected homogenate for cytokine analysis was cleared by centrifugation, and lysates were sterilized by filtration and stored at $-20^{\circ} \mathrm{C}$. A mixture of protease/oxygenation inhibitors $(0.2 \mathrm{mM}$ PMSF, $10 \mathrm{mg} / \mathrm{ml}$ aprotinin, $10 \mathrm{mg} / \mathrm{ml}$ leupeptin, $10 \mathrm{mg} / \mathrm{ml}$ pepstatin, and $0.5 \mathrm{mM}$ dithiothreitol) was added during preparation of organ samples to neutralize the effects of tissue proteases. Measurement of TNF- $\alpha$ levels was carried out using the TNF- $\alpha$ multiplex kit (Biosource/Invitrogen) according to the manufacturer's instructions.

\section{Virulence factor expression and virulence studies}

Capsule was measured by India Ink microscopy (29) and urease production by incubation on Christensen's agar (62), and the method of Liu et al. (63) was used to measure laccase activity. Virulence studies were conducted according to a previously described intravenous mouse meningoencephalitis model (64) using 10 Swiss Albino mice or an intranasal model using $10 \mathrm{CBA} / \mathrm{J}$ mice for each Cn strain (65). Fungal viability was confirmed by growth of inocula on YPD agar. Animal studies were approved by the Uni- versity of Illinois at Chicago Animal Care Committee. Studies involving human tissue were approved by the University of Illinois at Chicago Office for the Protection of Research Subjects and the Institutional Review Board (protocol \#2004-0600).

\section{Statistics}

Statistical significance of mouse survival times was assessed by KruskallWallis analysis (ANOVA on Ranks). Pairwise analyses were performed post hoc by using Dunn's procedure. Autophagic cell comparisons were performed by a $\chi^{2} 2$-sided test using a $2 \times 2$ contingency table. Fungal viability in macrophage cultures was compared by Fisher's exact test. Statistical analysis was conducted using GraphPad Prism software, version 4.03. In mouse mortality studies, time of death of the survivors was recorded as the day of experimental termination. Unless otherwise specified, all results are expressed as mean \pm SEM.

\section{Acknowledgments}

We would like to thank J. Moscat, P. Dennis, and G. Thomas for review of the manuscript. This work was supported in part by US Public Health Service Grant NIH-AI45995 and -AI49371. We also acknowledge the use of the C. neoformans Genome Project, Stanford Genome Technology Center (http://www-sequence.stanford.edu), funded by the NIAID/NIH under cooperative agreement AI47087.

Received for publication March 7, 2007, and accepted in revised form December 5, 2007.

Address correspondence to: Peter R. Williamson, University of Illinois at Chicago, Room 888, Building 910, m/c 735, $808 \mathrm{~S}$. Wood St., Chicago, Illinois 60612, USA. Phone: (312) 996-6070; Fax: (312) 413-1657; E-mail: prw@uic.edu.
1. Perfect, J.R., Wong, B., Chang, Y.C., Kwon-Chung, K.J., and Williamson, P.R. 1998. Cryptococcus neoformans: virulence and host defences. Med. Mycol. 36(Suppl. 1):79-86

2. Huffnagle, G.B., et al. 2000. Leukocyte recruitment during pulmonary Cryptococcus neoformans infection. Immunopharmacology. 48:231-236.

3. Feldmesser, M., Tucker, S., and Casadevall, A. 2001. Intracellular parasitism of macrophages by Cryptococcus neoformans. Trends Microbiol. 9:273-278.

4. Chun, C., Liu, O., and Madhani, H. 2007. A Link between virulence and homeostatic responses to hypoxia during infection by the human fungal pathogen Cryptococcus neoformans. PLOS Pathogens. 3:e22.

5. Panepinto, J., et al. 2005. The DEAD-box RNA helicase Vad1 regulates multiple virulence-associated genes in Cryptococcus neoformans. J. Clin. Invest. 115:632-641.

6. Waterman, S., et al. 2007. Role of a CUF1-CTR4 copper regulatory axis in the virulence of Cryptococcus neoformans. J. Clin. Invest. 117:794-802.

7. Burda, P., Padilla, S., Sarkear, S., and Emr, S. 2002. Retromer function in endosome-to-Golgi retrograde transport is regulated by the yeast Vps35 PtdIns 3-kinase. J. Cell Sci. 115:3889-3900.

8. Kihara, A., Noda, T., Ishihara, N., and Ohsumi, Y. 2001. Two distinct vps34 phohatidylinositol 3-kinase complexes function in autophagy and carboxyeptidase Y sorting in Sacharomyces cerevisiae. J. Cell Biol. 152:519-530.

9. Kametaka, S., Okano, T., Ohsumi, M., and Ohsumi, Y. 1998. Apg14p and Apg6/Vps30p form a protein complex essential for autophagy in the yeast, Saccharomyces cerevisiae. J. Biol. Chem. 273:22284-22291.

10. Ichimura, Y., et al. 2000. A ubiquitin-like system mediates protein lipidation. Nature. 408:488-492.

11. Noda, T., and Ohsumi, Y. 1998. Tor, a phosphati- dylinositol kinase homologue, controls autophagy in yeast. J. Biol. Chem. 273:3963-3966.

12. Ma, L., Chen, Z., Erdjument-Bromage, H., Tempst, P., and Pandolfi, P. 2005. Phosphorylation and functional inactivation of TSC2 by Erk: Implications for tuberous sclerosis and cancer pathogenesis. Cell. 121:179-193.

13. van Slegtenhorst, M., Carr, E., Stoyanova, R., Kruger, W.D., and Henske, E.P. 2004. Tsc1 and tsc2 regulate arginine uptake and metabolism in Schizosaccharomyces pombe. J. Biol. Chem. 279:12706-12713.

14. Shelton, S., et al. 2003. Saccharomyces cerevisiae contains a type II phosphoinositide 4-kinase. Biochem. J. 371:533-540.

15. Chen, H., Salopek, T., and Jimbow, K. 2001. The role of phosphoinositide 3-kinase in the sorting and transport of newly synthesized tyrosinaserelated protein-1 (TRP-1). J. Investig. Dermatol. Symp. Proc. 6:105-114.

16. Herman, P., and Emr, S. 1990. Characterization of VPS34, a gene required for vacuolar protein sorting and vacuole segregation in Saccharomyces cerevisiae. Mol. Cell. Biol. 10:6742-6754.

17. Liu, X., Hu, G., Panepinto, J., and Williamson, P. 2006. Role of a VPS41 homolog in starvation response and virulence of Cryptococcus neoformans. Mol. Microbiol. 61:1132-1146.

18. Pukkila-Worley, R., et al. 2005. Transcriptional network of multiple capsule and melanin genes governed by the Cryptococcus neoformans cyclic AMP cascade. Eukaryot. Cell. 4:190-201.

19. Moyrand, F., Klaproth, B., Himmelreich, U., Dromer, F., and Janbon, G. 2002. Isolation and characterization of capsule structure mutant strains of Cryptococcus neoformans. Mol. Microbiol. 45:837-849.

20. Lortholary, O., et al. 1999. Cytokine profiles of AIDS patients are similar to those of mice with dis- seminated Cryptococcus neoformans infection. Infect. Immun. 67:6314-6320.

21. Herring, A.C., Lee, J., McDonald, R.A., Toews, G.B., and Huffnagle, G.B. 2002. Induction of interleukin-12 and gamma interferon requires tumor necrosis factor alpha for protective T1-cell-mediated immunity to pulmonary Cryptococcus neoformans infection. Infect. Immun. 70:2959-2964.

22. Lindell, D., Ballinger, M., McDonald, R., Toews, G., and Huffnagle, G. 2006. Diversity of the T-cell response to pulmonary Cryptococcus neoformans infection. Infect. Immun. 74:4538-4548.

23. Feldmesser, M., Kress, Y., Novikoff, P., and Casadevall, A. 2000. Cryptococcus neoformans is a facultative intracellular pathogen in murine pulmonary infection. Infect. Immun. 68:4225-4237.

24. Henry-Stanley, M., Garni, R., and Wells, C. 2004. Adaptation of FUN-1 and calcofluor white stains to assess the ability of viable and nonviable yeast to adhere to and be internalized by cultured mammalian cells. J. Microbiol. Methods. 59:289-292.

25. Gutierrez, M., et al. 2004. Autophagy is a defense mechanism inhibiting BCG and Mycobacterium tuberculosis survival in infected macrophages. Cell. 119:753-766.

26. Fan, W., Kraus, P., Boily, M., and Heitman, J. 2005. Cryptococcus neoformans gene expression during murine macrophage infection. Eukaryot. Cell. 4:1420-1433.

27. Lang, T., et al. 1998. Aut2p and Aut7p, two novel microtubule-associated proteins are essential for delivery of autophagic vesicles to the vacuole. $E M B O J$. 17:3597-3607.

28. Kirisako, T., et al. 1999. Formation process of autophagosome is traced with Apg8/Aut7p in yeast. J. Cell Biol. 147:435-446.

29. Erickson, T., et al. 2001. Multiple virulence factors 
of Cryptococcus neoformans are dependent on VPH1. Mol. Microbiol. 42:1121-1131.

30. Guthrie, B., and Wickner, W. 1988. Yeast vacuoles fragment when microtubules are disrupted. J. Cell Biol. 107:115-120.

31. Kim, J., Huang, W., Stromhaug, P., and Klionsky, D. 2002. Convergence of multiple autophagy and cytoplasm to vacuole targeting components to a perivacuolar membrane compartment prior to de novo vesicle formation. J. Biol. Chem. 277:763-773.

32. Shin, Y., Kim, K., and Paik, Y. 2005. Alterations of protein expression in macrophages in response to Candida albicans infection. Mol. Cells. 20:271-279.

33. Liu, L., Tewari, R.P., and Williamson, P.R. 1999. Laccase protects Cryptococcus neoformans from antifungal activity of alveolar macrophages. Infect. Immun. 67:6034-6039.

34. Mednick, A.J., Nosanchuk, J.D., and Casadevall, A. 2005. Melanization of Cryptococcus neoformans affects lung inflammatory responses during cryptococcal infection. Infect. Immun. 73:2012-2019.

35. Randhawa, H.S., Mussa, A.Y., and Khan, Z.U. 2001. Decaying wood in tree trunk hollows as a natural substrate for Cryptococcus neoformans and other yeast-like fungi of clinical interest. Mycopathologia. 151:63-69.

36. Racicot, T.A., and Bulmer, G.S. 1985. Comparison of Media for the Isolation of Cryptococcus neoformans. Appl. Environ. Microbiol. 50:548-549.

37. Levine, B., and Klionsky, D.J. 2004. Development by self-digestion: molecular mechanisms and biological functions of autophagy. Dev. Cell. 6:463-477.

38. Cuervo, A. 2004. Autophagy: in sickness and in health. Trends Cell Biol. 14:70-77.

39. Shintani, T., and Klionsky, D.J. 2004. Autophagy in health and disease: a double-edged sword. Science. 306:990-995.

40. Amer, A., and Swanson, M. 2005. Autophagy is an immediate macrophage response to Legionella pnewmophila. Cell. Microbiol. 7:765-778.

41. Palmer, G., Kelly, M., and Sturtevant, J. 2007. Autophagy in the pathogen Candida albicans. Microbiology. 153:51-58.

42. Kitanovic, A., et al. 2005. Phosphatidylinositol 3-kinase VPS34 of Candida albicans is involved in filamentous growth, secretion of aspartic proteases, and intracellular detoxification. FEMS Yeast Res. 5:431-439.

43. Monari, C., et al. 2005. Cryptococcus neoformans capsu- lar glucuronoxylomannan induces expression of fas ligand in macrophages. J. Immunol. 174:3461-3468.

44. Deretic, V., et al. 2006. Mycobacterium tuberculosis inhibition of phagolysosome biogenesis and autophagy as a host defence mechanism. Cell. Microbiol. 8:719-727.

45. Barchiesi, F., Colombo, A.L., McGough, D.A., and Rinaldi, M.G. 1994. Comparative study of broth macrodilution and microdilution techniques for in vitro antifungal susceptibility testing of yeasts by using the National Committee for Clinical Laboratory Standards' proposed standard. J. Clin. Microbiol. 32:2494-2500.

46. National Committee on Clinical and Laboratory Standards. 2002. Reference method for broth dilution antifungal susceptibility testing of yeasts; approved standard - second edition. NCCLS document M27-A2. http://www.nccls.org/source/ orders/free/m27-a2.pdf.

47. Dannaoui, E., et al. 2007. Results obtained with various antifungal susceptibility testing methods do not predict early clinical outcome in patients with cryptococcosis. Antimicrob. Agents Chemother. 50:2464-2470.

48. Zhu, X., and Williamson, P.R. 2003. A CLC-type chloride channel gene is required for laccase activity and virulence in Cryptococcus neoformans. Mol. Microbiol. 50:1271-1281.

49. Varma, A., Edman, J.C., and Kwon-Chung, K.J. 1992. Molecular and genetic analysis of URAS transformants of Cryptococcus neoformans. Infect. Immun. 60:1101-1108.

50. Cox, G.M., Toffaletti, D.L., and Perfect, J.R. 1996. Dominant selection system for use in Cryptococcus neoformans. J. Med. Vet. Mycol. 34:385-391.

51. Storz, P., Doppler, H., Wernig, A., Pfizenmaier, K., and Muller, G. 1999. Cross-talk mechanisms in the development of insulin resistance of skeletal muscle cells palmitate rather than tumour necrosis factor inhibits insulin-dependent protein kinase B (PKB)/Akt stimulation and glucose uptake. Eur. J. Biochem. 266:17-25.

52. Bain, J., Stubberfied, C., and Gow, N. 2001. Urastatus-dependent adhesion of Candida albicans mutants. FEMS Microbiol. Lett. 204:323-328.

53. Panepinto, J., and Williamson, P. 2006. Intersection of fungal fitness and virulence in Cryptococcus neoformans. FEMS Yeast Res. 6:489-498.

54. Tucker, S.C., and Casadevall, A. 2002. Replica- tion of Cryptococcus neoformans in macrophages is accompanied by phagosomal permeabilization and accumulation of vesicles containing polysaccharide in the cytoplasm. Proc. Natl. Acad. Sci. U. S. A. 99:3165-3170

55. Levitz, S., DiBenedetto, D., and Diamond, R. 1987. A rapid fluorescent assay to distinguish attached from phagocytized yeast particles. J. Immunol. Methods. 101:37.

56. Kozel, T.R., Pfrommer, G.S., Guerlain, A.S., Highison, B.A., and Highison, G.J. 1988. Strain variation in phagocytosis of Cryptococcus neoformans: dissociation of susceptibility to phagocytosis from activation and binding of opsonic fragments of C3. Infect. Immun. 56:2794-2800.

57. Zhu, X., Gibbons, J., Garcia-Rivera, J., Casadevall, A., and Williamson, P.R. 2001. Laccase of Cryptococcus neoformans is a cell wall-associated virulence factor. Infect. Immun. 69:5589-5596.

58. Varma, A., and Kwon-Chung, K.J. 1991. Rapid method to extract DNA from Cryptococcus neoformans. J. Clin. Microbiol. 29:810-812.

59. Mizushima, N. 2004. Methods for monitoring autophagy. Int. J. Biochem. Cell Biol. 36:2491-2502.

60. Zhang, S., et al. 2006. The Hsp70 member, Ssa1 acts as a DNA-binding transcriptional co-activator in Cryptococcus neoformans. Mol. Microbiol. 62:1090-1101.

61. Noverr, M.C., Williamson, P.R., Fajardo, R.S., and Huffnagle, G.B. 2004. CNLAC1 is required for extrapulmonary dissemination of Cryptococcus neoformans but not pulmonary persistence. Infect. Immun. 72:1693-1699.

62. Cox, G., Mukherjee, J., Cole, G., Casadevall, A., and Perfect, J. 2000. Urease as a virulence factor in experimental cryptococcosis. Infect. Immun. 68:443-448.

63. Liu, L., Wakamatsu, K., Ito, S., and Williamson, P.R. 1999. Catecholamine oxidative products, but not melanin, are produced by Cryptococcus neoformans during neuropathogenesis in mice. Infect. Immun. 67:108-112.

64. Salas, S.D., Bennett, J.E., Kwon-Chung, K.J., Perfect, J.R., and Williamson, P.R. 1996. Effect of the laccase gene CNLAC1, on virulence of Cryptococcus neoformans. J. Exp. Med. 184:377-386.

65. Cox, G., et al. 2003. Superoxide dismutase influences the virulence of Cryptococcus neoformans by affecting growth within macrophages. Infect. Immun. 71:173-180 